\title{
The Mass Distribution in the Galactic Centre Estimated from OH/IR stars
}

\author{
M. Lindqvist, A.Winnberg, Onsala Space Observatory \\ H.J. Habing, Sterrewacht Leiden \\ H.E. Matthews, James Clerk Maxwell Telescope \\ F.M. Olnon, Sterrenwacht Dwingeloo
}

\begin{abstract}
The preliminary results of a search for OH/IR stars in the Galactic Centre using the VLA are presented. The goal of this project is to determine the mass density distribution from a few parsecs up to about $120 \mathrm{pc}$ of the Galactic Centre using stellar kinematics. Up to now, $125 \mathrm{OH} / \mathrm{IR}$ stars have been found. The distribution of the stars is clearly elongated with the major axis parallel to the galactic equator. The stars show a rotation of $117 \mathrm{~km} \mathrm{~s}^{-1} \mathrm{deg}^{-1}$ with a dispersion of $90 \mathrm{~km} \mathrm{~s}^{-1}$. Two preliminary models have been applied in order to determine the enclosed mass, resulting in $M(r)=4.0 \cdot 10^{6}$ $\cdot r^{1.4} \mathrm{Mo}$, where $r$ is the galactocentric distance in parsecs.
\end{abstract}

\section{Introduction}

The information on the mass distribution in the Galactic Centre comes mainly from the gas kinematics and IR radiation (cf. Genzel and Townes, 1987). However, the gas kinematics seems to be affected by explosive events and possible magnetic fields and is consequently not under the influence of pure gravitation. The problem with the 2 micron continuum radiation is the uncertain assumption about the mass/light ratio and interstellar absorption.

In contrast, the stellar kinematics should reflect the gravitational potential only and stars are therefore the most suitable probes to study the dynamics of the central parts of our Galaxy (cf. Sellgren et al., 1987; McGinn et al., these proceedings). We have made a survey of stars close the Galactic Centre in order to determine the mass density distribution from a few parsecs up to about $120 \mathrm{pc}$ galactocentric distances. The picture on this scale is unclear since the massive molecular clouds in this region have large non-circular (50-100 $\left.\mathrm{km} \mathrm{s}^{-1}\right)$ velocities. Inside the central parsec the number of detected OH/IR stars in this survey is too small to test the hypothesis of a central black hole (Lynden-Bell and Rees, 1971). The type of stars we have used are the so-called $\mathrm{OH} / \mathrm{IR}$ stars, which are at the end of their evolution and therefore probably constitute a dynamically relaxed system (cf. Herman and Habing, 1985, for a review of the nature of OH/IR stars). They are believed to be long-period variables situated at the top of the Asymptotic Giant Branch (AGB) in the H-R diagram (cf. Iben, 1983, for a review of 503 


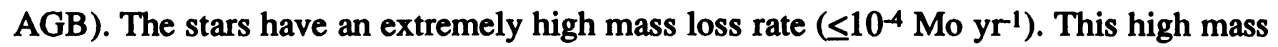
loss rate creates a circumstellar shell with such high dust content that the star cannot be observed at optical wavelengths any longer. At radio frequencies, three masing molecules have been found in the circumstellar environment of an OH/IR star: OH (cf. Eder et al., 1988), $\mathrm{H}_{2} \mathrm{O}$ (cf. Engels et al., 1986; Nyman et al., 1986) and SiO (cf. Jewell et al., 1985; Lindqvist et al., 1987a). The strong maser emisson and the characteristic spectra of the $1612 \mathrm{MHz} \mathrm{OH}$ line have given radio astronomy an excellent probe not only to study the mass distribution in the Galactic Centre but also to determine the distance to it (J. Cohen et al., these proceedings). Today, over $1000 \mathrm{OH} / \mathrm{IR}$ stars have been detected in the Galaxy (Olofsson, 1989) and they will constitute an excellent sample to study the large-scale dynamics and structure of our Galaxy. In fact, one $\mathrm{OH} / \mathrm{IR}$ star has been detected at $1612 \mathrm{MHz}$ in the LMC (Wood et al., 1986), and more detections in extemal galaxies will provide us with an important tool to determine distances, and hence with a new step on the distance ladder. In addition, OH/IR stars are important for our understanding of mass loss and hence of the chemical evolution of the Galaxy.

\section{Observations and Data Reduction}

The observations were made with the Very Large Array (VLA) in New Mexico using the spectral-line system at $1612 \mathrm{MHz}$ during 1984 and 1985 . Six fields were observed and the positions are given in Lindqvist et al. (1987b). Each field was observed with three frequency settings, centred on $+145,0,-145 \mathrm{~km} \mathrm{~s}^{-1}$ with respect to the Local Standard of Rest. The spectral band of each setting was divided into 128 channels with a resolution of $7.3 \mathrm{kHz}\left(1.4 \mathrm{~km} \mathrm{~s}^{-1}\right)$, resulting in a velocity coverage of $+/-217 \mathrm{~km} \mathrm{~s}^{-1}$ for each field. The reason that we used the VLA rather than a single dish telescope, was to resolve out the extended continuum background sources. This was possible by restricting ourselves to baselines longer than $900 \mathrm{~m}$. In this way we eliminated strong interstellar $\mathrm{OH}$ absorption lines that cause severe confusion in singledish data. A single dish survey for $\mathrm{OH} / \mathrm{IR}$ stars in the Galactic Centre was carried out with the $100-\mathrm{m}$ telescope. The results of this survey are presented in Habing et al. (1983).

The data have been reduced at the Onsala Space Observatory using the AIPS package. We have made maps of all the fields with $1024 \times 1024$ pixels, each pixel being 2.5" (total number of maps: 2304), and cleaned those maps containing strong sources. The synthesized beam was typically $8 " \mathrm{x} 6 "$ and the sensitivity $30 \mathrm{mJy}(3 \sigma)$. The data have then been put into cubes, 108 per field. The data cubes have been searched automatically for three consecutive channels with a flux higher than $50 \mathrm{mJy}$. To qualify as an $\mathrm{OH} / \mathrm{IR}$ star this signal must have a counterpart within $+/-60 \mathrm{~km} \mathrm{~s}^{-1}$ at the same position within the error.

\section{Results}

The number of OH/IR stars that we have found so far in the six fields is 125 including the 33 stars found by Winnberg et al. (1985). We still have some tentative detections which need further investigation. The distribution of the stars on the sky is 


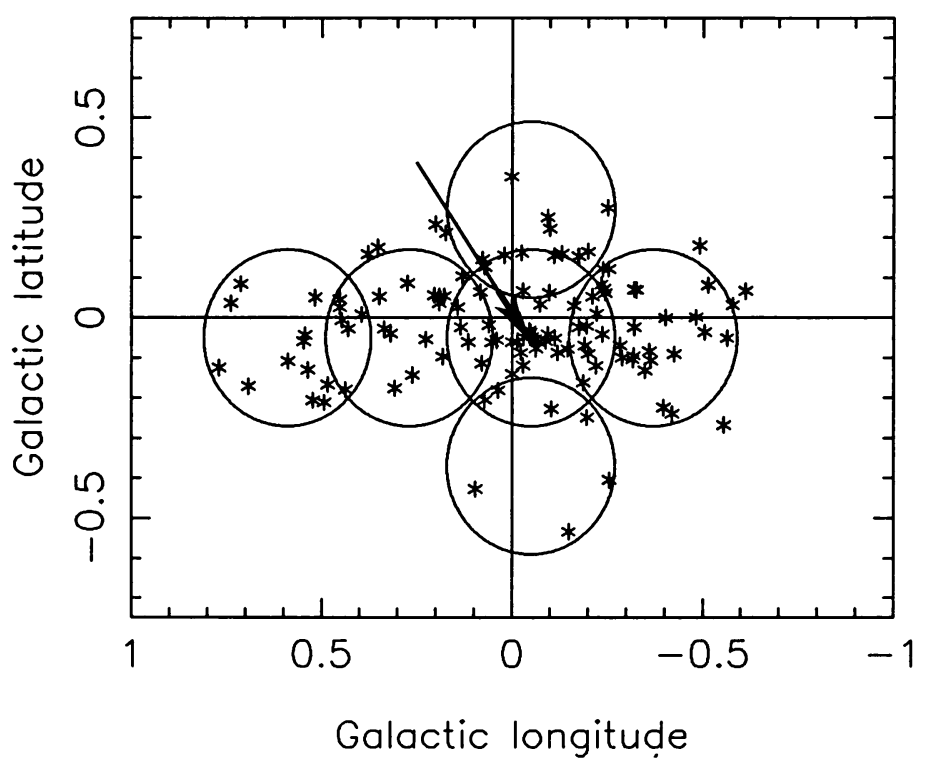

Fig. 1 The distribution of $125 \mathrm{OH} / \mathrm{IR}$ stars on the sky. The circles delineate the half-power loci of the VLA primary beams for the six fields mapped. The position of the radio source $\mathrm{Sgr} \mathrm{A}$ West is marked with an arrow.

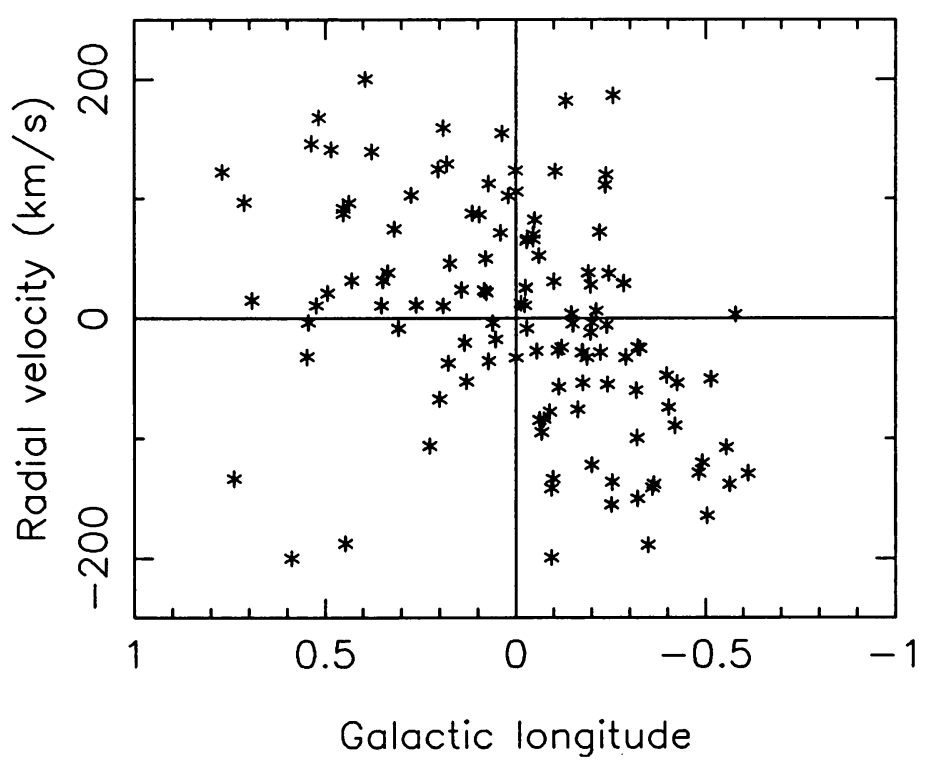

Fig. 2 The radial velocity of the $\mathrm{OH} / \mathrm{IR}$ stars as function of galactic longitude. A linear regression passes through the origin and has a slope of $117 \mathrm{~km} \mathrm{~s}^{-1} \mathrm{deg}^{-1}$. The velocity dispersion is $90 \mathrm{~km} \mathrm{~s}^{-1}$. 
shown in Figure 1. The circles delineate the half-power loci of the six primary beams of the VLA (HPBW= 27'). Our data cubes, however, cover 32' $x 32^{\prime}$. There is a clear increase in star density close to Sgr A West which is marked with an arrow. There is also clear evidence of an elongated distribution with the major axis parallell to the galactic equator. According to the distribution of stars in the 1-v diagram (Figure 2) we are dealing with a system of stars with rotation. A linear regression gives a net rotation of $117 \mathrm{~km} \mathrm{~s}^{-1} \mathrm{deg}^{-1}$, but the dispersion is as high as $90 \mathrm{~km} \mathrm{~s}^{-1}$.

\section{$\mathrm{OH} / \mathrm{IR}$ STARS}

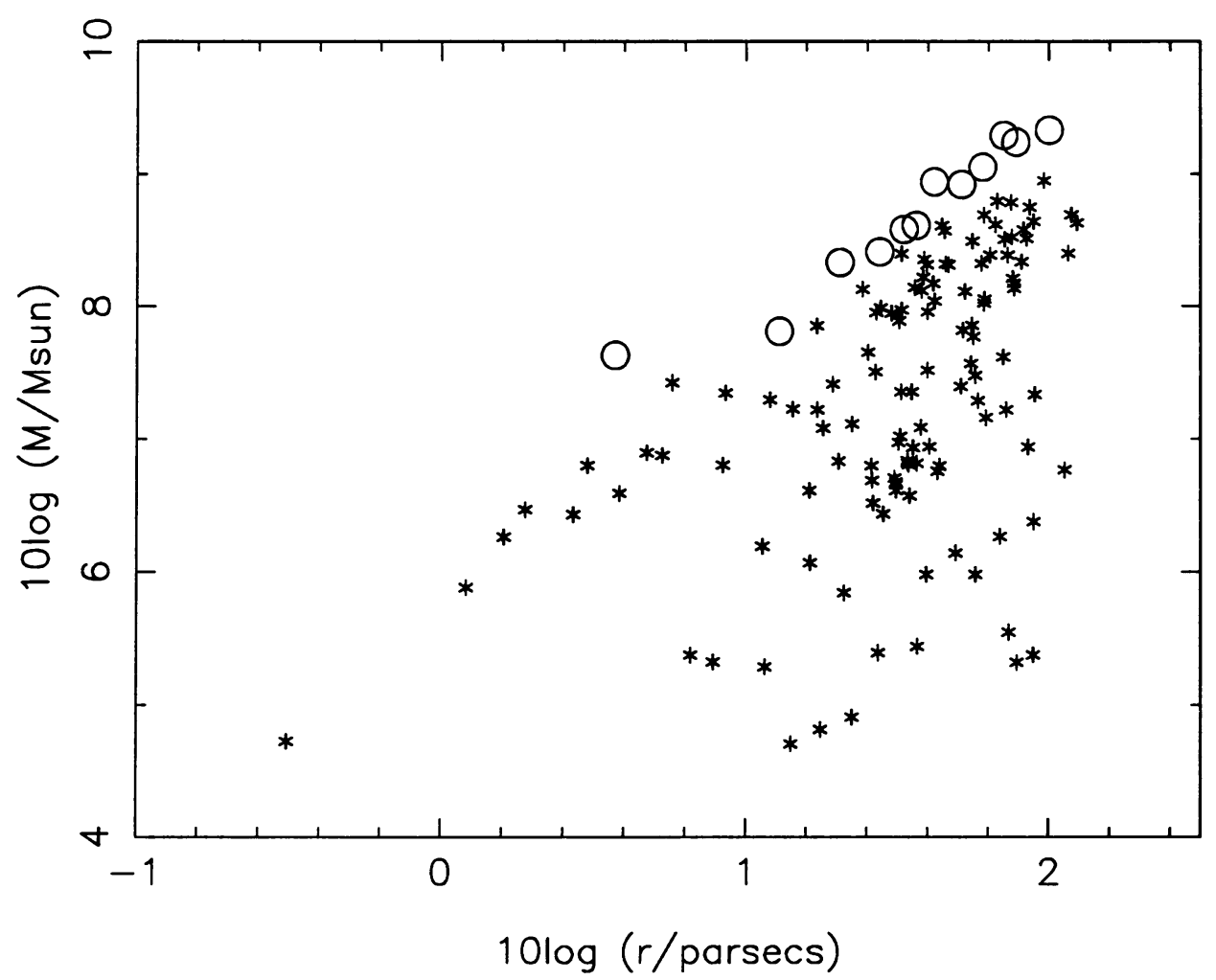

Fig. 3 Preliminary estimate of the enclosed mass (M) inside the galactocentric radius (r). A spherical mass distribution and circular orbits have been assumed. The values (depicted by the asterisks) are lower limits of the enclosed mass if we assume that the observed radial velocities are equal to the orbital velocities. The circles represent values calculated according to a method proposed by Bahcall and Tremaine (1981). 
As a crude preliminary analysis we have assumed a spherically symmetric mass distribution and circular orbits. The enclosed mass as a function of the projected galactocentric distance is shown in Figure 3. We have calculated the projected radius assuming a distance to the Galactic Centre of $8.5 \mathrm{kpc}$ and we have also assumed that the radial velocity of each source is the velocity in a circular orbit. The enclosed mass has then been evaluated using the formula:

$$
M(r)=v^{2} r / G
$$

where $\mathbf{G}$ is the gravitational constant. Each point is actually a lower limit to the enclosed mass since the true radius may be larger than the projected radius and the radial velocity only measures the line-of-sight component of the orbital velocity. An estimate of the enclosed mass would therefore be the upper envelope of these points. We have also used a method discussed by Bahcall and Tremaine (1981) called 'the projected mass method' using the formula:

$$
M_{I}(r)=24 /(\pi G N) \sum v_{i}{ }^{2} r_{i}
$$

The factor $24 / \pi$ is a correction for the distribution of eccentricities. It is here assumed that this distribution is somewhere between an isotropic distribution and a distribution consisting of only linear orbits. We have divided the 125 stars into 12 groups with approximately 10 stars in each group. The resulting mass distribution from these calculations is marked by the circles (Figure 3). A linear regression gives:

$$
<\mathrm{M}>=4.0 \cdot 10^{6} \mathrm{r}^{1.4}
$$

with $\langle\mathrm{M}\rangle$ in $\mathrm{Mo}$ and $\mathbf{r}$ in $\mathbf{p c}$.

We intend to build a more sophisticated model of the mass density distribution that will fit our data as well as possible. We will then introduce a flattended gravitational potential and take account of the velocity dispersion by modelling the distribution of orbital eccentricities.

In addition to the mass density distribution, our sample of $\mathrm{OH} / \mathrm{IR}$ stars is very useful for two reasons. The distances to the Galactic Centre can be determined using a method described by Cohen et al. (these proceedings). A monitoring project is now under way using the VLA for this purpose (van Langevelde, Goss, Habing, and Winnberg, in preparation). Secondly, the OH/IR stars are nearly all at the same distance from us. Therefore, they can be used to study luminosity functions of all kinds of radiation, such as $\mathrm{OH}, \mathrm{H}_{2} \mathrm{O}, \mathrm{SiO}$, and $\mathrm{IR}$ continuum. We have started to study the $\mathrm{SiO}$ (Lindqvist et al., 1987a) and the $\mathrm{H}_{2} \mathrm{O}$ masers (Lindqvist et al., 1989).

Acknowledgement This work is supported by the Swedish Natural Research Council (NFR). 


\section{References}

Bahcall, J.N., Tremaine, S.:1981, Astrophys. J., 244, 805

Eder, J., Lewis, B.M., Terzian, Y.:1988, Astrophys. J. Suppl. Ser., 66, 183

Engels, D., Schmid-Burgk, J., Walmsley, C.M.:1986, Astron. Astrophys. 167, 129

Genzel, R., Townes, C.H.:1987, Ann. Rev. Astron. Astrophys., 25, 377

Habing, H.J., Olnon, F.M., Winnberg, A., Matthews, H.E., Baud,B.:1983, Astron. Astroph., 128, 230

Herman, J., Habing, H.J.:1985, Phys. Rep., 124, No4, 255

Iben, I. Jr, Renzini, A.:1983, Ann. Rev. Astron. Astrophys., 21, 271

Jewell, P.R., Walmsley, C.M., Wilson, T.L., Snyder, L.E.:1985, Astrophys. J., 298, L55

Lynden-Bell, D., Rees, M.J.:1971, M.N.R.A.S., 152, 461

Lindqvist, M., Winnberg, A., Ukita, N., Johansson, L.E.B.:1987a, Astron. Astrophys., 172, L3

Lindqvist, M., Winnberg, A., Matthews, H.E., Habing, H.J., Olnon, F.M.: 1987b, In Late Stages of Stellar Evolution, eds S. Kwok and S.R. Pottasch, p79, Dordrecht: Reidel

Lindqvist, M., Winnberg, A., Forster, R.:1989, in prep

Nyman, L.-Å., Johansson, L.E.B., Booth, R.S.: 1986, Astron . Astrophys., 160,352

Olofsson, H.:1989, IAU Coll. No. 106, eds. Johnson, H.R., Zuckerman, B., Cambridge Univ. Press, in press

Sellgren, K., Hall, D.N.B., Kleinmann, S.G., Scoville, N.Z.:1987, Astrophys. J., 317, 881

Winnberg, A., Baud, B., Matthews, H.E., Habing, H.J., Olnon, F.M.:1985, Astrophys. J., 291, L45

Wood, P.R., Bessell, M.S., Whiteoak, J.B.,:1986, Astrophys. J., 306, L81 